\title{
Controlled sequential assembly of metal-organic polyhedra into colloidal gels with high chemical complexity
}

Min Ying Tsang, Shun Tokuda, Po-Chun Han, Zaoming Wang, Alexandre Legrand, Marina Kawano, Masahiko Tsujimoto, Yuki Ikeno, Kenji Urayama, Kevin C.-W. Wu, Shuhei Furukawa*

Dr. Min Ying Tsang, Shun Tokuda, Zaoming Wang, Dr. Alexandre Legrand, Marina Kawano, Masahiko Tsujimoto, Prof. Shuhei Furukawa

Institute for Integrated Cell-Material Sciences (WPI-iCeMS), Kyoto University, Yoshida,

Sakyo-ku, Kyoto 606-8501, Japan

E-mail: shuhei.furukawa@icems.kyoto-u.ac.jp

Zaoming Wang, Prof. Shuhei Furukawa

Department of Synthetic Chemistry and Biological Chemistry, Graduate School of

Engineering, Kyoto University, Katsura, Nishikyo-ku, Kyoto 615-8510, Japan

Shun Tokuda, Marina Kawano

Undergraduate School of Industrial Chemistry, Faculty of Engineering, Kyoto University,

Yoshida, Sakyo-ku, Kyoto 606-8501, Japan

Po-Chun Han, Prof. Kevin C.-W. Wu

Department of Chemical Engineering, Program of Green Materials and Precision Devices, National Taiwan University, Taipei 10617, Taiwan

Yuki Ikeno, Prof. Kenji Urayama

Department of Macromolecular Science and Engineering, Kyoto Institute of Technology,

Matsugasaki, Sakyo-ku, Kyoto 606-8585, Japan

Keywords: (coordination polymer particles, hierarchical structures, gels, metal-organic polyhedral, assembly)

\begin{abstract}
Assembling many chemical components into a material in a controlled manner is one of the biggest challenges in chemistry. Particularly porous materials with multivariate character within their scaffolds are expected to demonstrate synergistic properties. In this study, we show a synthetic strategy to construct porous networks with multiple chemical components. By taking advantage of the hierarchical nature of a colloidal system based on metal-organic polyhedra (MOPs), we developed a two-step assembly process to form colloidal networks; assembling of MOPs with the organic linker to the formation of MOP network as a colloidal particle, followed
\end{abstract}


by further connecting colloids by additional crosslinkers, leading to colloidal networks. This synthetic process allows not only for the use of different organic linkers for connecting MOPs and colloidal particles, respectively, but for assembling different colloidal particles formed by various MOPs. The proof-of-concept of this tuneable multivariate colloidal gel system offers an alternative to developing functional porous soft materials with multifunction.

\section{Introduction}

Increasing the chemical complexity by assembling different functional components is key for creating complex architectures with not only material properties beyond a single function but also tailorable characters. ${ }^{[1-3]}$ Such complex systems have attracted great interests particularly for crystalline and ordered porous solids such as metal-organic frameworks (MOFs) and covalent organic frameworks (COFs), in which more than two functional moieties (e.g., ligands with different functional groups or various metal clusters) can be integrated within the same structure $^{[4-6]}$ The chemical complexity, together with the decorated pore surface of these multivariate porous frameworks, showed significant benefits in developing functional materials for catalysis ${ }^{[7-10]}$, sensing ${ }^{[11]}$ and separation applications ${ }^{[12,13]}$. Yet, the lack of predictable arrangement of mixed compositions in the structure makes it difficult to control the positioning of the specific molecular components in the framework scaffold. ${ }^{[14,15]}$

The multivariate character of these materials is not limited to a single motif at the molecular level but can be integrated at longer length scales in order for the chemical complexity to emerge, for instance, via epitaxial growth of two distinct $\mathrm{MOF}^{[16,17]}$ or the control of the size and shape of MOF particles resulting to different layout of mesostructures (colloidal MOFs) and material behaviors (e.g., photonic or sensing property). ${ }^{[18-20]}$ Nevertheless, in the first case, it remains challenging to increase the chemical complexity by integrating various types of material due to the requirement for matching the lattice parameters at the crystal interface, while for the second one, the mesostructures of these types of assembled colloidal 
MOFs are usually not stable due to weak interactions, and it is still challenging to assemble several distinct nanocrystals into a single mesostructure.

To tackle these issues, one can consider using a colloidal gel system, in which chemically different mesoscale colloidal particles can be interconnected ${ }^{[21]}$ through electrostatic interaction or chemical bonding ${ }^{[22-25]}$ to form multicomponent colloidal networks. Interestingly, depending on the interaction between particles, the assembled structures of two chemically distinct colloids can either (i) be self-sorted into a network and form double interpenetrated colloidal networks, or (ii) lead to the preferential interaction between two different colloids (for instance, electrostatic interaction) yielding to the formation of a mixed colloidal network. ${ }^{26,27]}$ In recent years, a number of MOF particle-based gels have been reported, proving the possibility of creating new types of multicomponent colloidal gels with versatile functionalities. ${ }^{[28-31]}$ Metal-organic polyhedra (MOPs) with discrete cage structures present a good alternative to MOFs due to their processability in solution and lability. When used as secondary building blocks, MOPs can be assembled with additional linkers into colloidal particles or colloidal gel networks. ${ }^{[32-38]}$ However, all of these systems have been assembled from a single MOP component. Therefore, integration of more than two distinct components during the self-assembly process remains a major challenge due to the lack of methodology. 


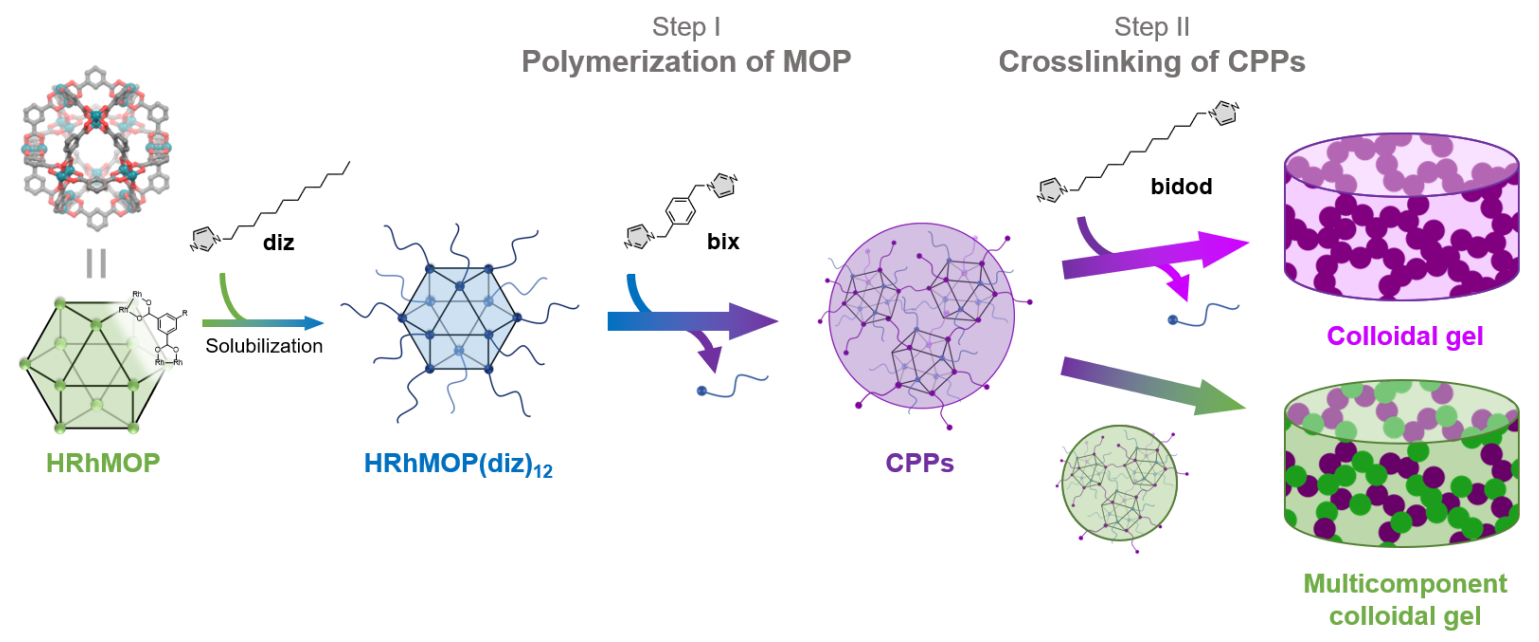

Figure 1. Schematic representation of the step-by-step hierarchical assembly from HRhMOP to colloidal gel via (i) HRhMOP solubilization in DMF by addition of diz ligand; (ii) CPPs formation via replacing diz with the $1^{\text {st }}$ addition of crosslinkers (bix, bibph or bidod); and (iii) CPP-based gel formation via the $2^{\text {nd }}$ addition of crosslinkers (bix, bibph or bidod).

Here, we show a coordination chemistry approach to form multicomponent hierarchical structures from interconnected MOPs. Our methodology takes advantage of available coordination sites exposed on the surface of the MOPs, where first a ditopic N-donor ligand leads to the formation of MOP networks as colloidal particles, which can be further crosslinked into a colloidal gel network by the addition of a secondary N-donor ligand. The transfer of MOPs to complex colloidal gel networks using this two-steps assembly strategy will demonstrate the feasibility to install selected functional moieties at the scales of the colloidal particles or colloidal gel networks (Figure 1). This will allow the precise engineering of the supramolecular structure and advance the multi-functionalities in a controlled manner.

\section{Results and discussion}

\subsection{Controlled synthesis of coordination polymer particle (CPP)}

In this study, we chose the stable rhodium-based MOP, $\left[\mathrm{Rh}_{2}(\mathrm{bdc})_{2}\right]_{12}(\mathbf{H R h M O P})$, assembled from 12 dirhodium acetate paddlewheels and 24 benzene-1,3-dicarboxylate (bdc) with cuboctahedral geometry for the formation of colloidal particles. ${ }^{[38]}$ By taking advantage of the reactivity of the external axial site of the rhodium paddlewheel, we previously demonstrated 
the polymerization of MOPs with bisimidazole derivatives such as 1,4-bis[(1H-imidazol-1yl)methyl]benzene (bix) as linkers. On the one hand, the gradual addition of the ditopic Ndonor ligand bix to the MOP solution resulted in the formation of colloidal spheres (named as coordination polymer particles, CPPs). On the other hand, the generation of kinetically-trapped

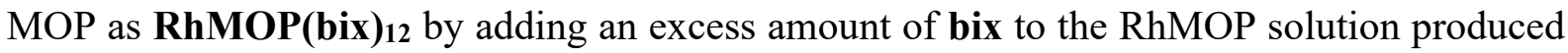
a colloidal gel network. Note that the latter strategy leads to the spontaneous formation of a colloidal gel network once the MOP and bix are mixed, which does not allow for further control of the assembly process, including its termination at the stage of colloidal formation. In order to achieve a multicomponent colloidal structure, one needs to use colloidal particles as initial building blocks. Here, we first optimized the reaction condition for the CPP formation based on HRhMOP with different types of ditopic N-donor ligands and various stoichiometric ratios. Then, we optimized the condition to connect these CPP with another bisimidazole crosslinker to form the colloidal gel network. Finally, two chemically distinct but isostructural CPPs (one with HRhMOP and the other with BrRhMOP) were mixed to yield multicomponent colloidal gel networks (BrRhMOP, $\left[\mathrm{Rh}_{2}(5-\mathrm{Br}-\mathrm{bdc})_{2}\right]_{12}, \quad 5$-Br-bdc $=$ 5-bromobenzene-1,3dicarboxylate).

A series of ditopic N-donor bisimidazole ligands, bix, 4,4'-bis(1H-imidazol-1ylmethyl)biphenyl (bibph), and 1,12-di(1H-imidazol-1-yl)dodecane (bidod) were selected as bridging linkers to sequentially connect MOPs or CPPs. In order to process HRhMOP in solution, the cage was solubilized according to a reported coordination solubilizer method, which requires the coordination of monodentate $N$-dodecylimidazole (diz) to all of the 12 axial sites of $\mathrm{Rh}$ paddlewheels exposed on the surface of the molecule to yield soluble $\operatorname{HRhMOP}(\operatorname{diz}){ }_{12}{ }^{[37]}$

Formation of CPPs was then investigated via sequential addition of 0.5 molar equivalents per step of three distinct bisimidazole linkers, bix, bibph, or bidod, into the DMF solution of HRhMOP(diz) 12. $^{[37]}$ Replacement of $\mathbf{~ d i z ~ b y ~ b i s i m i d a z o l e ~ l i n k e r s ~ ( L ) ~ i n i t i a t e s ~ t h e ~}$ 
linkage between $\mathbf{H R h M O P}(\mathbf{d i z})_{\mathbf{x}}(\mathbf{L})_{\mathbf{y}}$ cages and leads to a MOP network in the form of CPPs. The use of bix as a linker provided dispersible CPPs, while the bibph and bidod generated undesired agglomerates of CPPs, which make it difficult to be further processed (Figure S5). Therefore, a series of CPPs made with bix as linkers were selected for further investigation.

In the MOP network linked by bix, the HRhMOP acts as a node linked up, in principle, to 12 other MOPs. The effective connectivity of MOP can be estimated from the chemical composition of the CPP determined by proton nuclear magnetic resonance measurement $\left({ }^{1} \mathrm{H}\right.$ NMR, Figure S8) and represented by the branch functionality, $f$, a parameter that indicates the number of rhodium sites per MOP that are used to connect with neighboring MOPs (see Supplementary Information). ${ }^{[37,42,43]}$ In order to correlate the added bix quantity with the branch functionality, the corresponding CPPs were synthesized with different molar equivalents of bix linker (3, 4, 6 and 12 molar equivalents) added. The calculated $f$ increases up to 8 by the addition of 6 molar equivalents of bix but decreases by the addition of an excess amount of bix, which is most likely due to the coordination of bix as a mono-dentate fashion (Table 1). As further addition of bridging ligands is required to allow connections between CPPs to form a colloidal gel network, the obtained CPPs should have enough accessible coordinative sites at the exohedral HRhMOP. Therefore, the CPPs formed with 3 molar equivalent addition of bix and having a relatively low $f$ value were selected for further studies.

Table 1. Molar equivalents of bix added versus CPP composition and branch functionality $f$ analyzed by ${ }^{1} \mathrm{H}$ NMR measurements.

\begin{tabular}{ccc}
\hline molar eq. of bix added (per MOP) & molecular formula & branch functionality, $f$ \\
\hline 3 & $\operatorname{HRhMOP}(\mathbf{b i x})_{5.2}(\mathbf{d i z})_{4.9}$ & 3.8 \\
4 & $\operatorname{HRhMOP}(\mathbf{b i x})_{5.7}(\mathbf{d i z})_{2.9}$ & 6.9 \\
6 & $\operatorname{HRhMOP}(\mathbf{b i x})_{6.2}(\mathbf{d i z})_{1.8}$ & 8.0 \\
12 & $\operatorname{HRhMOP}(\mathbf{b i x})_{7.3}(\mathbf{d i z})_{1.5}$ & 6.3 \\
\hline
\end{tabular}




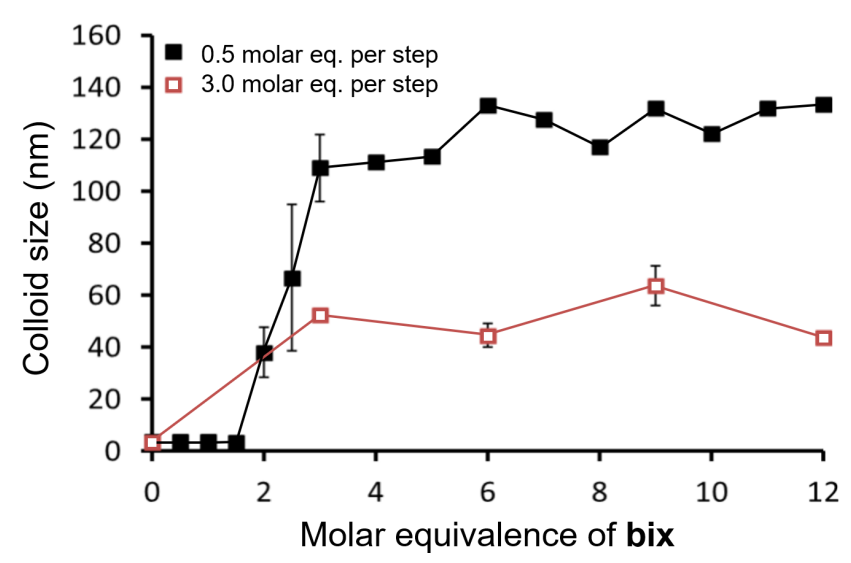

Figure 2. The average diameter of CPPs formed by the addition of bix from 0 to 12 molar equivalent measured by dynamic light scattering (DLS). 0.5 molar equivalents per step (black) and 3 molar equivalents per step (red) were investigated. The MOP concentration is $0.93 \mathrm{mM}$.

The size of CPP is expected to affect the macroporosity and connectivity of the corresponding colloidal gel networks. As reported previously, the CPP formation process can be tuned by the molar equivalent of bix added at each step, in which the first addition would generate nuclei and the following addition would contribute to particle growth. ${ }^{[36]}$ Hence, the effects of different concentrations of HRhMOP(diz $)_{12}$ and stepwise amount of bix added on the resulting CPP size were investigated by dynamic light scattering (DLS) titration experiments (Figure 2). When the addition of bix changed from 0.5 molar equivalents to 3.0 molar equivalents for each step, the size of final CPPs was decreased from $98 \pm 14 \mathrm{~nm}$ to $55 \pm$ $10 \mathrm{~nm}$ as also confirmed by scanning electron microscopy (SEM) images (Figure 3). This size reduction is attributed to the larger numbers of nuclei formed, yielding to smaller CPPs. In addition, the increase in the concentration of HRhMOP(diz) 12 from $0.93 \mathrm{mM}$ to $1.86 \mathrm{mM}$ raises the collision probability of particles, leading to a larger size of CPP $(189 \pm 30 \mathrm{~nm})$. In contrast, lowering the concentration to $0.46 \mathrm{mM}$ led to a smaller CPP particle size $(62 \pm 10 \mathrm{~nm})$. 


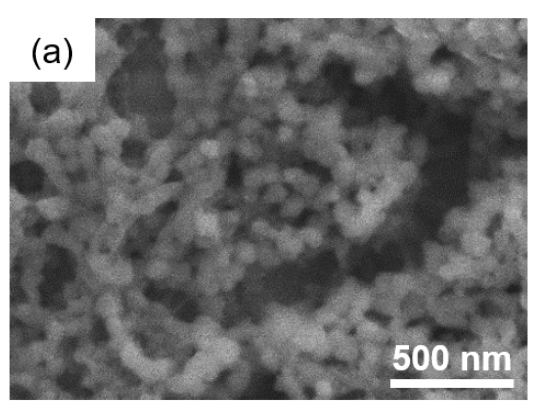

(b)

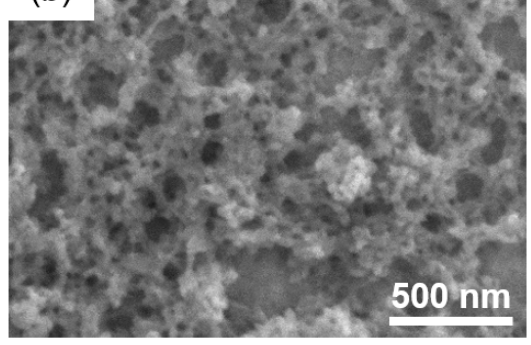

(c)

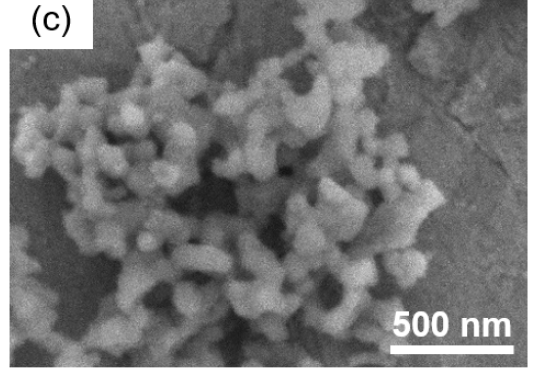

(d)

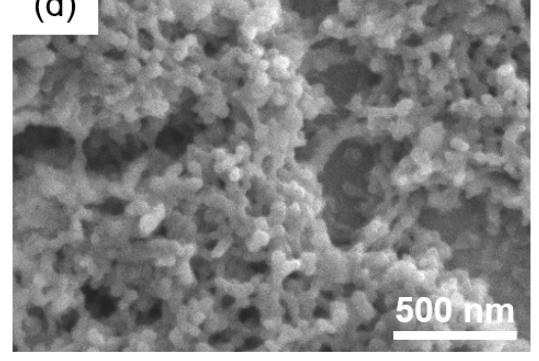

Figure 3. SEM images of CPPs formed under different synthetic conditions (a) 0.5 eq. bix/step,

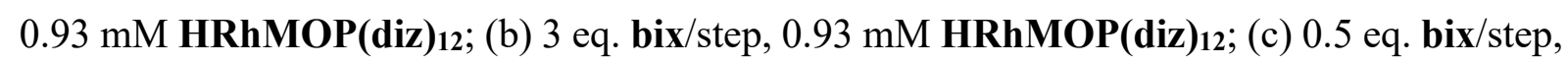

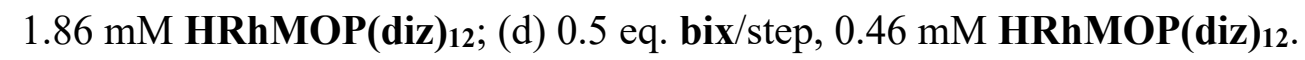

\subsection{Investigation of colloidal gel formation from CPPs}

In order to demonstrate the possibility of forming colloidal gels with CPPs, two types of CPPs with different sizes were produced by different addition rates of bix (CPP-large: $98 \pm 14 \mathrm{~nm}$ and CPP-small: $55 \pm 10 \mathrm{~nm}$ ) were selected for further investigations. We firstly attempted the gelation with various concentrations of CPP-large $(4 \mathrm{mg} / \mathrm{mL}, 8.5 \mathrm{mg} / \mathrm{mL}, 17 \mathrm{mg} / \mathrm{mL}$, and 42 
$\mathrm{mg} / \mathrm{mL}$ in DMF) by adding 12 molar equivalents bix and reacting at $80{ }^{\circ} \mathrm{C}$ for $2 \mathrm{~h}$. Note that the molar equivalence of added bix here was calculated as a relative molar ratio to HRhMOP in the CPP, which was estimated from the weight and ${ }^{1} \mathrm{H}$ NMR spectra. Only the high concentration of CPP-large (42 mg/mL) formed corresponding gel, but the CPP with lower concentrations gave suspensions (Figure S10).

To gain more insight into the gelation mechanism, the gelation process of $42 \mathrm{mg} / \mathrm{mL}$ CPP-large and CPP-small, after the addition of 12 molar equivalents of bix, were monitored by time-resolved dynamic light scattering (TRDLS) measurements at $50{ }^{\circ} \mathrm{C} .{ }^{[36-37,44]}$ The timeaveraged scattering light intensity $\langle I\rangle_{T}$ was found to be almost constant at the early stage of the reaction before fluctuating at 38 min for CPP-large (Figure 4a). This random fluctuation is a clear indication of the sol to gel transition due to the loss of ergodicity during the percolation of colloidal particles. Insight into the gelation mechanism can be extrapolated from the timeevolution of the intensity correlation functions (ICFs), $g^{(2)}(\tau)-1$, which is a function measuring the similarity between scattering light signal at different delay times (Figure 4b). Indeed, ICFs were fitted by a single stretched exponential function and give access to the following parameters:

$$
g^{(2)}(\tau)-1=\sigma_{\mathrm{I}}^{2}\left[\exp \left\{-\left(\frac{\tau}{\tau_{f}}\right)^{\beta}\right\}\right]^{2}
$$

where $\sigma_{\mathrm{I}}^{2}$ is the initial amplitude of the ICF, $\tau_{f}$ is the relaxation time related to the diffusion rate of particles, and $\beta$ is the stretched exponent that reflects the distribution of $\tau_{f}$. $\tau_{f}$ is further related to the correlation length $\xi$, which reflects the evolution of the density of the network during the reaction time in the solution phase, through Equation 2: 


$$
\xi=\frac{q^{2} k_{B} T}{6 \pi \eta} \tau_{f}
$$

where $q, k_{B}, T, \eta$ are the scattering vector, the Boltzmann constant, the absolute temperature, and the viscosity of media, respectively (see the Supplementary Information for the derivation of Equation 2). In the first $10 \mathrm{~min}$ of the reaction, $\tau_{f}$ and the corresponding correlation length $\xi$ were almost constant, indicating that the CPPs were individually diffusing and not aggregating with each other (Figures $4 \mathrm{c}$ and $\mathrm{S} 13 \mathrm{~h}$ ). This was also proven by the values of $\beta$ close to unity $(\approx 0.9)$, which reflects the relatively narrow size distribution of the CPP as observed by the SEM measurements. After this induction period, $\tau_{f}$ increased while $\beta$ decreased toward the gelation point. This simultaneous change of $\tau_{f}$ and $\beta$ indicates that the average particle size and size distribution are no anymore uniform, which is most likely explained by the stochastic aggregation of colloidal particles and the formation of a gel network. This agrees with the drastic increase of the correlation length $\xi$, (Figure S13h). The TRDLS experiment on CPPsmall also showed a similar trend to the CPP-large as shown in Figure S13.

(a)

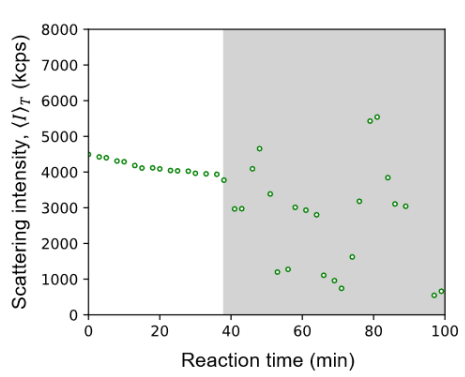

(b)

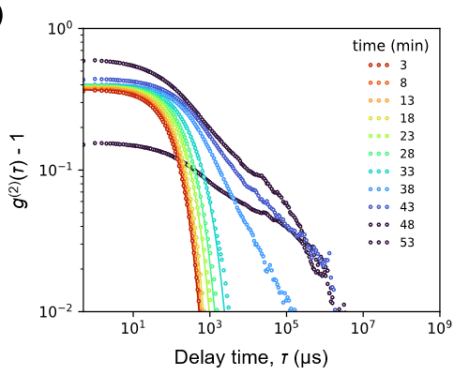

(c)

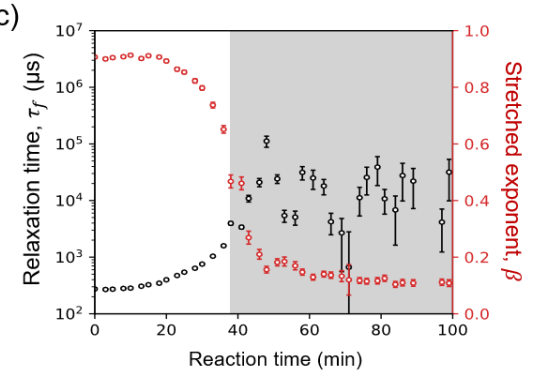

Figure 4. Gelation process of CPP-large at $50{ }^{\circ} \mathrm{C}$ monitored by TRDLS measurement. (a)

Time-averaged scattering light intensity as a function of the reaction time. (b) ICFs as a function of the delay time for different reaction times. Corresponding fitting curves are shown only for data points before the gelation point. (c) Evolution of fitting parameters $\tau_{f}$ and $\beta$ as a function of the reaction time. The gray region highlights the effect of the frozen mobility of particles on the DLS response due to gel formation. 
Differences in gelation time as a function of temperatures were also observed by TRDLS measurements. At $50{ }^{\circ} \mathrm{C}$, the sol-gel transition for CPP-small occurs at $107 \mathrm{~min}$ after the addition of bix. In contrast, the gel was formed in $19 \mathrm{~min}$ for the same system heated at 80 ${ }^{\circ} \mathrm{C}$ (Figure S12). This is most likely caused by the increase in the diffusion rate of particles and the kinetics of ligand exchange reaction accompanying the colloidal aggregation when increasing the temperature. In addition, the gelation time is dependent on the CPP size as seen from the gelation thresholds of 107 min and 38 min measured for CPP-small and CPP-large, respectively at $50{ }^{\circ} \mathrm{C}$ (Figure S13). This can be explained by the difference of van der Waals interaction between colloidal particles, which is estimated to be proportional to the particle diameter from Hamaker's theory. ${ }^{[45]}$ These examples illustrate the possibility of controlling gelation time by changing reaction temperature and CPP size, while retaining the chemical composition of the resulting colloidal gel network. Moreover, the reduction of the number of bix added from 12 to 6 eq. led to an increase in the gelation time (Figure S14), which is most likely attributed to the reduced coordinative interactions between CPPs that hindered the gelation process. For the purpose of accumulating more data points during the gelation process, the gelation reaction at $50{ }^{\circ} \mathrm{C}$ is used for the following investigation. To further confirm that the CPP gelation is induced by the second addition bix, a control experiment was performed using TRDLS to follow the reaction of CPP-large without the addition of bix (Figure S14). CPPlarge suspension showed almost constant $\tau_{f}$ and $\beta$ during the $6 \mathrm{~h}$ of heating at $50{ }^{\circ} \mathrm{C}$. This indicates that CPP is stable itself in DMF and gelation is indeed driven by crosslinking of CPPs mediated by bix molecules.

This successful assembly via two-step addition of bix to form CPP-based gel envisaged incorporating different crosslinkers (bibph and bidod) in a second step. However, only the addition of bidod to more than $42 \mathrm{mg} / \mathrm{mL}$ CPP-large was able to form a gel under the synthetic condition of $80^{\circ} \mathrm{C}$ for $2 \mathrm{~h}$. In contrast, the addition of bibph gave a suspension after the $2 \mathrm{~h}$ reaction at $80{ }^{\circ} \mathrm{C}$ (Figure $\mathrm{S} 15$ ). We attributed this difference in gelation to the variation in 
crosslinking reactivity of the linkers as: bidod $>$ bix $>$ bibph, which was proven by the difference of gelation time observed by TRDLS (Figure S16). Therefore, to investigate the multivariate colloidal gel, the system with CPP-large as cores and bidod as secondary linkers was selected and its gelation condition was optimized to obtain more mechanically durable colloidal gel.

(a)

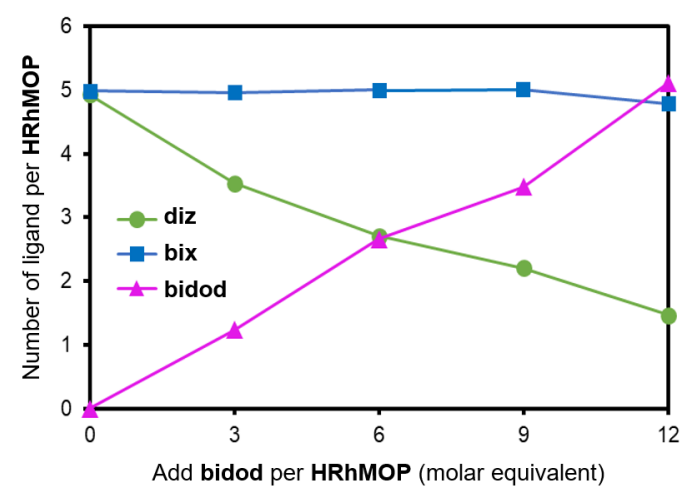

(b)

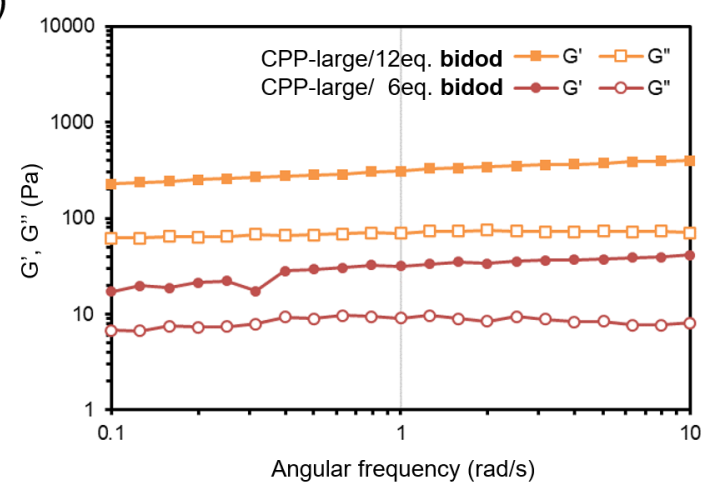

Figure 5. Investigation of the different equivalent of bidod for colloidal gel formation. (a) composition of linkers (diz, bix and bidod) per cage measured by ${ }^{1} \mathrm{H}$ NMR. (b) Angular frequency dependence of linear dynamic shear moduli for CPP-large/bidod gels with 6 and 12 equivalents of bidod conducted under $25^{\circ} \mathrm{C}$ with a strain amplitude of $0.2 \%$. 

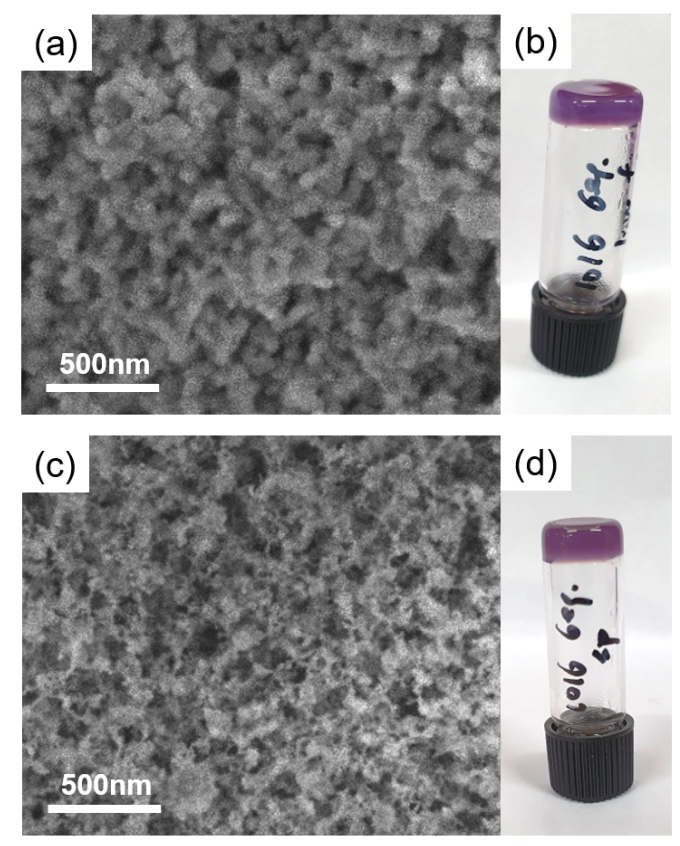

Figure 6. SEM images of the corresponding aerogels and photographs of obtained wet gels (a,b) CPP-large/6 eq. bidod gel. (c,d) CPP-small/6 eq. bidod gel. The corresponding aerogels were obtained from the wet gels via supercritical $\mathrm{CO}_{2}$ treatment (see Supporting Information).

It is expected that the strength of the gels is predominantly affected by the addition of bidod due to the cross-linkage, interaction between CPPs, and the chemical environment. To clarify the effect of crosslinker amount on the strength of the gels, a series of CPP-large/bidod gels with addition of 3, 6, 9, 12 molar equivalents bidod have been synthesized (Figure S18) from which the compositions were determined by ${ }^{1} \mathrm{H}$ NMR spectroscopy (Figure S19). The average number of diz, bix, and bidod per HRhMOP was estimated using the average integration of selected representative signals (Figure 5a). Interestingly, the relative number of bix molecules to HRhMOP remained almost the same compared to the original CPP itself regardless of the quantity of bidod added. In contrast, the relative number of diz linearly decreased and that of bidod linearly increased as a function of the amount of bidod added. This clearly indicates that bix and HRhMOP molecules are forming a stable coordination network inside CPPs and newly added bidod can only replace diz molecules in CPP. Unlike the CPP 
formation, branch functionality $f$ is no longer suitable to reflect the connectivity between CPPs since bidod can crosslink MOPs within the same CPP as well as with the neighboring CPPs. Therefore, the relation between the quantity of bidod addition and the stiffness of the gels was further investigated by rheology measurements (Figures 5b and S20). The CPP-large/bidod gels were prepared in situ in the rheometer by heating the mixture of CPP-large suspension and 6 or 12 equivalents bidod at $80{ }^{\circ} \mathrm{C}$ for $20 \mathrm{~min}$, followed by determination of the storage $\left(G^{\prime}\right)$ and loss $\left(G^{\prime \prime}\right)$ modulus at $25^{\circ} \mathrm{C}$. The $G^{\prime}$ was always higher than $G$ ', and they were almost constant independently of the shearing frequency and strain, which demonstrate their linear elastic property. It was found that the G' and G'” of CPP-large/12 eq. bidod gel are greater than those of the CPP-large/6 eq. bidod gel, which agrees with the increased number of bidod present in the gel network. This result further confirms that the addition of bidod enhances the crosslinking between CPPs and forms a more robust gel. Furthermore, the successful formation of gels from CPP-small with either bix or bidod was confirmed (Figures 6 and S13), which illustrates the possibility of altering the network structures.

\subsection{Multivariate colloidal assembly}

In order to demonstrate the versatility of material composition, isostructural MOPs, with a substitution at $5^{\text {th }}$ position of benzene-1,3-dicarboxylate with hydrophilic functionality $(-\mathrm{OH})$, OHRhMOP, and with an electron-rich functionality (-Br), BrRhMOP are studied. The corresponding CPP-OH and CPP-Br are synthesized following the CPP-large protocol (addition of totally 3 molar equivalents bix; 0.5 molar equivalents per step at $25^{\circ} \mathrm{C}$ ), to yield spherical colloids (CPP-OH and CPP-Br) from both $0.93 \mathrm{mM}$ DMF solution of OHRhMOP(diz) $)_{12}$ and BrRhMOP(diz) 12 with particle sizes of $115 \pm 5 \mathrm{~nm}$ and $47 \pm 7 \mathrm{~nm}$, respectively (Figure S 21a). Second step addition of 6 molar equivalents of bidod into 42 $\mathrm{mg} / \mathrm{mL} \mathbf{C P P}-\mathbf{O H}$ and CPP-Br suspended in DMF also led to the formation of the 
corresponding colloidal gels (Figure S21b), proving the feasibility to vary the functional group in the step of CPPs.

To demonstrate the possibility to create a multivariate colloidal gel network formed from the mixing of CPP-Br with 'pristine' CPP based on HRhMOP, the presence of $\mathrm{Br}$ component in the colloidal aerogel network was mapped via energy dispersive X-ray analysis (EDX) in transmission electron microscope (TEM) due to its electron-rich property (Figure 7). The EDX image in Figure 7a shows a homogeneous distribution of $\mathrm{Br}$ (green) and $\mathrm{Rh}$ (red) atoms in the system, which is also confirmed by a 1:1 ratio of $\mathrm{Br}$ and $\mathrm{Rh}$ from the elemental composition analysis (Figure S22c). Then the possibility to increase the complexity of this sequential assembly system was illustrated by mixing CPP-large and CPP-Br. For proof-ofconcept, a hybrid CPP gel was synthesized by mixing CPP-large and CPP-Br with a 1 to 1.2 ratio (estimated $\mathrm{Br}: \mathrm{Rh}=0.45: 1)$ and the addition of 6 molar equivalents of bidod. A relatively weak gel was formed after heating the mixture at $80{ }^{\circ} \mathrm{C}$ for $2 \mathrm{~h}$. Its corresponding aerogel was prepared via supercritical $\mathrm{CO}_{2}$ drying and analyzed by TEM and EDX. Figures 7e-h clearly show that the $\mathrm{Br}$ element is segregated from the $\mathrm{Rh}$ one, thus demonstrating a nonhomogeneous distribution of CPP-large and CPP-Br in the system. The elemental composition of $\mathrm{Br}$ and $\mathrm{Rh}$ analyzed by EDX was almost identical to the expected molar ratio of the mixtures of CPP-large and CPP-Br $(\mathrm{Br}: \mathrm{Rh}=0.45: 1)$ (Figure S22d). This result further demonstrates the possibility of incorporating different CPPs during the stepwise formation of the colloidal gel network. 

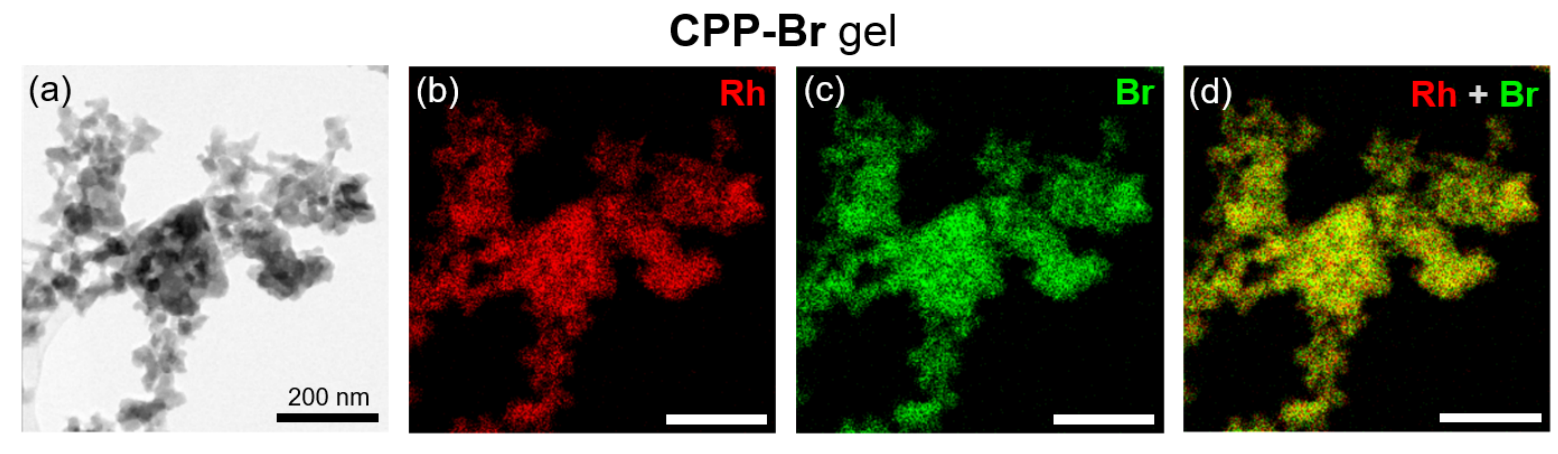

\section{1:1.2 CPP-large/CPP-Br gel}
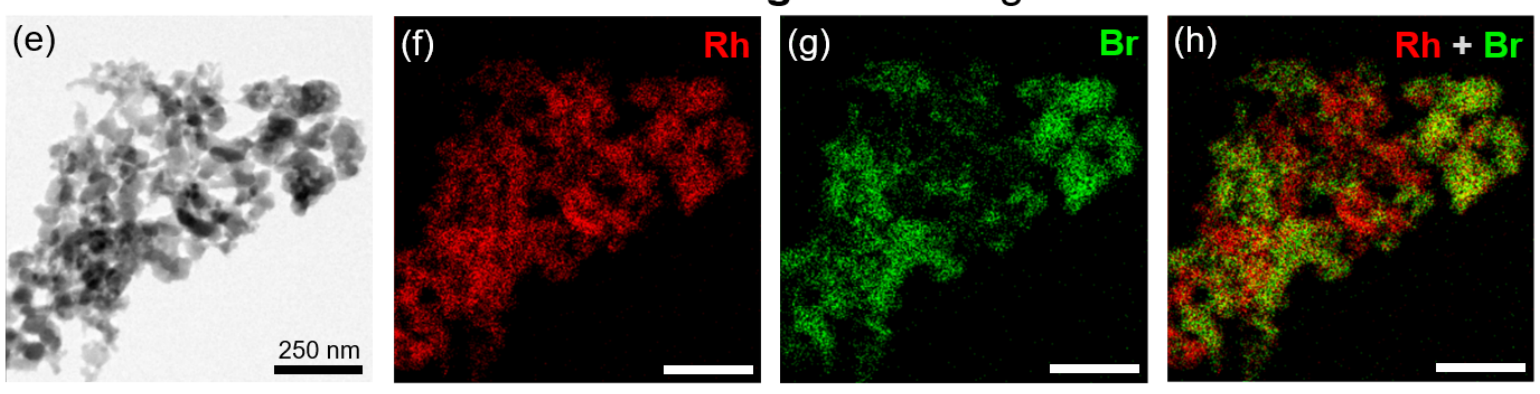

Figure 7. TEM images and the corresponding EDX of the as-made (a-d) CPP-Br gel and (e-h)

CPP-Br/CPP-large (selected elements: Br (green), Rh (red)).

\section{Conclusion}

The two-step hierarchical construction strategy developed here has allowed us to fine-tune the properties of the colloidal gel network via the control of the CPP size, gel composition (CPP core and its connectivity of gel network) and crosslinking degree as demonstrated by the combination of TRDLS, ${ }^{1} \mathrm{H}$ NMR and rheology techniques. In addition, the possibility to i) have control over the colloidal size without altering the core components, ii) modify the mesoscale connectivity with different crosslinkers and iii) install functional moieties via alteration of RhMOPs and CPPs open up a new alternative to develop multivariate colloidal gel via hierarchical assembly to form multi-functional materials for potential applications such as sensing and separation.

\section{Supporting Information}

Supporting Information is available from the Wiley Online Library or from the author. 
Acknowledgments

M. Y. T is grateful to the Japan Society for the Promotion of Science (JSPS) for Post-Doctoral Fellowships. This study was supported by JSPS KAKENHI Grant Number 20K15366 (Wakate) for A. L. and 19H04575 (Coordination Asymmetry) and 18H01995 (Kiban B) for S.F. K. C.W. W. and P. H. acknowledge the Ministry of Science and Technology, Taiwan (MOST 1102628-E-002-014) for the financial support. The authors thank Prof. Susumu Kitagawa, Dr. Arnau Carne-Sanchez, Dr. Gavin Craig for their fruitful discussions. The authors also thank the iCeMS Analysis Center for access to analytical instruments.

Received: ((will be filled in by the editorial staff))

Revised: ((will be filled in by the editorial staff)) Published online: ((will be filled in by the editorial staff))

\section{References}

[1] A. L. Goodwin, Nat. Commun., 2019, 10, 4461.

[2] H. S. Azevedo, S. L. Perry, P. A. Korevaar, D. Das, Nat. Chem., 2020, 12, 793.

[3] Z. Hassan, Y. Matt, S. Begum, M. Tsotsalas, S. Brase, Adv. Funct. Mater., 2020, 30, 1907625.

[4] H. Furukawa, U. Muller, O. M. Yaghi, Angew. Chem. Int. Ed., 2015, 54, 3417.

[5] L. Feng, K-Y. Wang, G. S. Day, H-C. Zhou, Chem. Soc. Rev., 2019, 48, 4823.

[6] J. Jiao, W. Gong, X. Wu, S. Yang, Y. Cui, Coord. Chem. Rev., 2019, 385, 174.

[7] X-S. Wang, C-H. Chen, F. Ichihara, M. Oshikiri, J. Liang, L. Li, Y. Li, H. Song, S. Wang, T. Zhang, Y-B. Huang, R. Cao, J. Ye, App. Cat. B: Envir., 2019, 253, 323.

[8] S. Patial, P. Raizada, V. Hasija, P. Singh, V. Kumar Thakur, V-H. Nguyen, Mater. Today Energy, 2021, 19, 100589.

[9] B. Lerma,-Berlanga, C. R. Ganivet, N. Almora-Barrios, S. Tatay, Y. Peng, J. Albero, O.

Fabelo, J. Gonzalez-Platas, H. Garcia, N. M. Padial, C. Marti-Gastaldo, J. Am. Chem. Soc., 2021, 143, 1798.

[10] Y. Wang, H. Lv, E.S. Grape, C. A. Gaggioli, A. Tayal, A. Dharanipragada, T. Willhammar, A. K. Inge, X. Zou, B. Liu, Z. Huang, J. Am. Chem. Soc., 2021, 143, 6333.

[11] Q. Zhang, M. Wahiduzzaman, S. Wang, S. Henfling, N. Ayoub, E. Gkaniatsou, F. Nouar, C. Sicard, C. Martineau, Y. Cui, G. Maurin, G. Qian, C. Serre, Chem, 2019, 5(5), 1337 
[12] W. Fan, S. Yuan, W. Wang, L. Feng, X. Liu, X. Zhang, X. Wang, Z. Kang, F. Dai, D. Yuan, D. Sun, H-C. Zhou, J. Am. Chem. Soc., 2020, $142(19), 8728$.

[13] M. Yang, P. Guo, X. Feng, W. Zhang, G. Yang, Micro. \& Meso. Mater., 2021, 324, 111297.

[14] W. Xu, B. Tu, Q. Liu, Y. Shu, C-C. Liang, C. S. Diercks, O. M. Yaghi, Y-B. Zhang, E. Deng, Q. Li, Nat. Rev. Mater., 2020, 5, 764.

[15] A. Dutta, Y. Pan, J-Q. Liu, A. Kumar, Coord. Chem. Rev., 2021, 445, 214074.

[16] S. Furukawa, K. Hirai, K. Nakagawa, Y. Takashima, R. Matsuda, T. Tsuruoka, M. Kondo, R. Haruki, D. Tanaka, H. Sakamoto, S. Shimomura, O. Sakata, S. Kitagawa, Angew. Chem. Int. Ed., 2009, 48, 1766.

[17] O. Kwon, J. Y. Kim, S. Park, J. H. Lee, J. Ha, H. Park, H. R. Moon, J. Kim, Nat. Commun., 2019, 10, 3620.

[18] N. Yanai, S. Granick, Angew. Chem. Int. Ed., 2012, 51, 5638.

[19] C. Avci, M. L. D. Marco, C. Byun, J. Perrin, M. Scheel, C. Boissiere, M. Faustini, Adv. Mater., 2021, 2104450, 1.

[20] Y. Liu, J. Wang, I. Imaz, D. Maspoch, J. Am. Chem. Soc., 2021, 143, 12943.

[21] C. F. Cordova, E. D. Gado, G. Foffi, M. Bouzid, Soft Matter, 2020, 16, 4414.

[22] J. Li, W. Y. Wong, X. M. Tao, Nanoscale, 2020, 12, 1281.

[23] C. T. Dersire, A. Lotierzo, R. D. Arrua, E. F. Hilder, S. A. F. Bon, Green Chem., 2018, $20,2499$.

[24] J. Fu, J. Polym. Sci. B Polym. Phys., 2018, 56, 1336.

[25] S. K. Nair, S. Basu, B. Sen, M-H. Lin, A. N. Kumar, Y. Yuan, P. L. Cullen, D. Sarkar, Scientific Reports, 2019, 9, 1072.

[26] H. Wang, M. B. Hansen, D. W. P. M. Lowik, J. C. M. van Hest, Y. Li, J. A. Jansen, S. C. G. Leeuwenburgh, Adv. Mater., 2011, 23, H119.

[27] P. Rusch, D. Zambo, N. C. Bigall, Acc. Chem. Res., 2020, 53, 2414. 
[28] J. J. Marrero-Tellado, D. D. Diaz, CrystEngComm., 2015, 17, 7978.

[29] B. Bueken, N. V. Velthoven, T. Willhammar, T. Stassin, I. Stassen, D. A. Keen, G. V. Baron, J. F. M. Denayer, R. Ameloot, S. Bals, D. D. Vos, T. D. Bennett, Chem. Sci., 2017, 8, 3939.

[30] J. Hou, A. F. Sapnik, T. D. Bennett, Chem. Sci., 2020, 11, 310.

[31] Z. Zhuang, Z. Mai, T. Wang, D. Liu, Coord. Chem. Rev., 2020, 421, 213461.

[32] D. J. Tranchemontagne, Z. Ni, M. O’Keeffe, O. M. Yaghi, Angew. Chem. Int. Ed., 2008, $47,5136$.

[33] A. Legrand, G. A. Craig, M. Bonneau, S. Minami, K. Urayama, S. Furukawa, Chem. Sci., 2019, 10, 10833.

[34] A. Legrand, L. Liu, P. Royla, T. Aoyama, G. A. Craig, A. C. Sanchez, K. Urayama, J. J. Weigand, C. Lin, S. Furukawa, J. Am. Chem. Soc., 2021, 143, 3562.

[35] Z. Wang, G. A. Craig, A. Legrand, F. Haase, S. Minami, K. Urayama, S. Furukawa, Chem. Asian. J., 2021, DOI: 10.1002/asia.202100080.

[36] A. C. Sanchez, G. A. Craig, P. Larpent, T. Hirose, M. Higuchi, S. Kitagawa, K. Matsuda, K. Urayama, S. Furukawa, Nat. Commun., 2018, 9, 2506.

[37] A. C. Sanchez, J. Albalad, T. Grancha, I. Imaz, J. Juanhuix, P. Larpent, S. Furukawa, D. Maspoch, J. Am. Chem. Soc., 2019, 141, 4094.

[38] A. C. Sanchez, G. A. Craig, P. Larpent, V. Guillerm, K. Urayama, D. Maspoch, S. Furukawa, Angew. Chem. Int. Ed., 2019, 58, 6347.

[39] S. Furukawa, N. Horike, M. Kondo, Y. Hijikata, A. C. Sanchez, P. Larpent, N. Louvain, S. Diring, H. Sato, R. Matsuda, R. Kawano, S. Kitagawa, Inorg. Chem., 2016, 55, 10843

[40] R. Kawano, N, Horike, Y. Hijikata, M. Kondo, A. C. Sanchez, P. Larpent, S. Ikemura, T. Osaki, K. Kamiya, S. Kitagawa, S. Takeuchi, S. Furukawa, J. Chempr., 2017, 2(3), 393.

[41] S. Mollick, S. Fajal, S. Mukherjee, S. K. Ghosh, Chem. Asian. J., 2019, 14, 3096. 
[42] S. E. Shim, S. Yang, M-J. Jin, Y. H. Chang, S. Choe, Colloid Polym. Sci., 2004, 283, 41.

[43] J. R. Moon, J. H. Kim, Bull. Korean. Chem. Soc., 2006, 27(12), 1981.

[44] M. Shibayama, T. Norisuye, BCSJ, 2002, 75, 641.

[45] H. C. Hamaker, Physica. 1937, 4, 1058. 